\title{
STRONGER LASOTA-YORKE INEQUALITY FOR ONE-DIMENSIONAL PIECEWISE EXPANDING TRANSFORMATIONS
}

\author{
PEYMAN ESLAMI AND PAWEL GÓRA \\ (Communicated by Bryna Kra)
}

\begin{abstract}
For a large class of piecewise expanding $\mathcal{C}^{1,1}$ maps of the interval we prove the Lasota-Yorke inequality with a constant smaller than the previously known $2 / \inf \left|\tau^{\prime}\right|$. Consequently, the stability results of Keller-Liverani apply to this class and in particular to maps with periodic turning points. One of the applications is the stability of acim's for a class of W-shaped maps. Another application is an affirmative answer to a conjecture of Eslami-Misiurewicz regarding acim-stability of a family of unimodal maps.
\end{abstract}

\section{INTRODUCTION}

The problem of stability in general and the stability of invariant measures in particular are one of the most important (and difficult) questions in dynamical systems. Here, we are concerned with the stability of absolutely continuous invariant measures (acim-stability) for piecewise expanding maps of an interval. The general setting is as follows.

Definition 1.1 (acim-stability). Given a family of maps $\left\{\tau_{\epsilon}: X \rightarrow X\right\}_{\epsilon \geq 0}$ with corresponding invariant densities $\left\{f_{\epsilon}\right\}_{\epsilon \geq 0}$, we say that $\tau_{0}$ is acim-stable if $\lim _{\epsilon \rightarrow 0} \tau_{\epsilon}=$ $\tau_{0}$ implies $\lim _{\epsilon \rightarrow 0} f_{\epsilon}=f_{0}$. The limits are taken with respect to properly chosen metrics on the space of maps and densities, respectively.

A relevant notion of closeness for maps under consideration is convergence in the Skorokhod metric (see Definition 4.4), and for the corresponding invariant densities, in this paper it is convergence in $\mathcal{L}^{1}$.

Stability problems were investigated in a multitude of works; most relevant to our study are [6] and [7.

The main motivation for this work was to prove acim-stability for some W-shaped maps with slopes $>1$ (by a slope we shall always mean the absolute value of the slope). A troublesome property of such maps is that they contain periodic turning points. Let us consider such a map $W$ with a fixed turning point $p_{0}$. This would not be a problem if $\left|W^{\prime}\right|>2$ (whenever the derivative exists). In fact, then the acim-stability of $W$ follows directly from the results of [6]. However, if $1<\left|W^{\prime}\right| \leq 2$ near $p_{0}$, the standard procedure, which is to work with an iterate of $W$ that has

Received by the editors October 6, 2011 and, in revised form, January 7, 2012 and February 1, 2012.

2010 Mathematics Subject Classification. Primary 37A10, 37A05, 37E05.

The first author was supported by the INdAM-COFUND Marie Curie Fellowship during the final stages of the preparation of this article. 
derivative $>2$, fails due to the presence of the fixed turning point $p_{0}$. We bypass this problem by deriving a stronger Lasota-Yorke inequality, hence avoiding the iteration of the maps.

A. Lasota and J. Yorke [10] first discovered this inequality and used it to prove the existence of acim's for piecewise expanding $\mathcal{C}^{2}$ maps. Z. Kowalski [8 later proved the existence of acim's for piecewise expanding $\mathcal{C}^{1,1}$ maps.

In this work we consider piecewise expanding $\mathcal{C}^{1,1}$ maps of an interval (see [9] for higher-dimensional results) 1 We prove a Lasota-Yorke inequality with a constant which is smaller than the previously known $2 / \inf \left|\tau^{\prime}\right|$ for a fairly large class of maps. This allows us to apply the stability theorems of $[7$. One of the implications would be the acim-stability of a class of maps in the presence of periodic turning points.

We point out that perhaps one may be able to enlarge the class of maps under consideration to piecewise expanding $\mathcal{C}^{1+\epsilon}$ maps (i.e. with a Hölder condition on the derivatives). However, there are examples of piecewise expanding $\mathcal{C}^{1}$ maps with no acim's, as shown in [5].

\section{Setting AND NOtATion}

Suppose $I=[0,1]$ and $m$ is the Lebesgue measure on $I$. We will be concerned with piecewise expanding $\mathcal{C}^{1,1}$ maps on $I$, which are defined as follows.

Definition 2.1 (Piecewise expanding $\mathcal{C}^{1,1}$ functions). Suppose there exists a partition $\mathcal{P}=\left\{I_{i}:=\left(a_{i-1}, a_{i}\right), i=1, \ldots, q\right\}$ of $I$ such that $\tau: I \rightarrow I$ satisfies the following conditions. For all $i$,

(1) $\tau_{i}:=\tau_{\left.\right|_{i}}$ is monotonic, $\mathcal{C}^{1}$, and can be extended to the closed interval $\left[a_{i-1}, a_{i}\right]$ as a $\mathcal{C}^{1}$ function;

(2) $\tau_{i}^{\prime}$ is Lipschitz, i.e., there exists a constant $M_{i}$ such that $\left|\tau_{i}^{\prime}(x)-\tau_{i}^{\prime}(y)\right| \leq$ $M_{i}|x-y|$, for all $x, y \in I_{i}$;

(3) $\left|\tau_{i}^{\prime}(x)\right| \geq s_{i}>1$ for all $x \in I_{i}$.

Then, we say $\tau \in \mathcal{T}(I)$, the class of piecewise expanding $\mathcal{C}^{1,1}$ maps on $I$.

If a family of maps $\left\{\tau_{\epsilon}\right\}$ satisfies the above conditions with uniform constants $s_{i}$ and $M_{i}$ (i.e. independent of $\epsilon$ ), then we shall write $\left\{\tau_{\epsilon}\right\} \subset \mathcal{T}(I)$ uniformly.

We will use the following notation throughout the paper.

Let

$$
s:=\min _{1 \leq i \leq q} s_{i} \text { and } \quad M:=\max _{1 \leq i \leq q} M_{i}
$$

Also, let

$$
\delta_{i}^{ \pm}:=\delta_{\left\{\tau\left(a_{i}^{ \pm}\right) \notin\{0,1\}\right\}}= \begin{cases}0 & \text { if } \tau\left(a_{i}^{ \pm}\right) \in\{0,1\}, \\ 1 & \text { if } \tau\left(a_{i}^{ \pm}\right) \notin\{0,1\},\end{cases}
$$

where $\tau\left(a_{i}^{ \pm}\right)$means $\lim _{x \rightarrow a_{i}^{ \pm}} \tau(x)$. For example, $\delta_{i}^{+}=1$ means that the left endpoint of the $(i+1)$-st branch of $\tau$ is hanging (doesn't touch 0 or 1 ).

\footnotetext{
${ }^{1}$ Upon a more detailed look at the approach of Liverani in 9], we believe that his approach, at least in the piecewise $C^{2}$ case, essentially leads to the same stronger Lasota-Yorke inequality which is presented in this paper.
} 
We denote by $P_{\tau}$ the Perron-Frobenius operator induced by $\tau$ on $\mathcal{L}^{1}(I)$. That is,

$$
P_{\tau} f=\sum_{y \in \tau^{-1}(x)} g(y) f(y)=\sum_{i=1}^{q} \frac{f\left(\tau_{i}^{-1}(x)\right)}{\left|\tau^{\prime}\left(\tau_{i}^{-1}(x)\right)\right|} \chi_{\tau\left(a_{i-1}, a_{i}\right)}(x),
$$

where $g(y):=1 /\left|\tau^{\prime}(y)\right|$. Note that $\sup _{I_{i}}|g| \leq 1 / s_{i}<1$.

The (total) variation of a function $f: I \rightarrow \mathbb{R}$ is defined as

$$
\bar{\bigvee}_{I} f=\sup _{0=x_{0}<x_{1}<x_{2}<\cdots<x_{N}=1} \sum_{i=1}^{N}\left|f\left(x_{i}\right)-f\left(x_{i-1}\right)\right|,
$$

where the supremum is taken over all the partitions of the interval $I$.

The essential variation of a function $f: I \rightarrow \mathbb{R}$ is defined by

$$
\bigvee_{I} f=\inf _{g \simeq f} \bar{\bigvee}_{I} g
$$

where $\simeq$ denotes equality almost everywhere with respect to Lebesgue measure.

We will consider $P_{\tau}$ on the space of functions of the bounded essential variation

$$
B V(I)=\left\{f \in \mathcal{L}^{1}(I): \bigvee_{I} f<\infty\right\}
$$

modulo equality almost everywhere, with the norm

$$
\|f\|_{B V}=\|f\|_{\mathcal{L}^{1}}+\bigvee_{I} f
$$

Since functions of the bounded variation are continuous except at the most countable number of points at which they have one-sided limits, we assume that functions in $B V(I)$ satisfy

$$
f\left(x_{0}\right)=\max \left\{\lim _{x \rightarrow x_{0}^{-}} f(x), \lim _{x \rightarrow x_{0}^{+}} f(x)\right\}
$$

at any point $x_{0} \in I$. For such functions $\bigvee_{I} f=\bar{\bigvee}_{I} f$. For more information about $B V(I)$ we refer the reader to [1] and [3].

\section{LASOTA-YORKE INEQUALITY}

Let

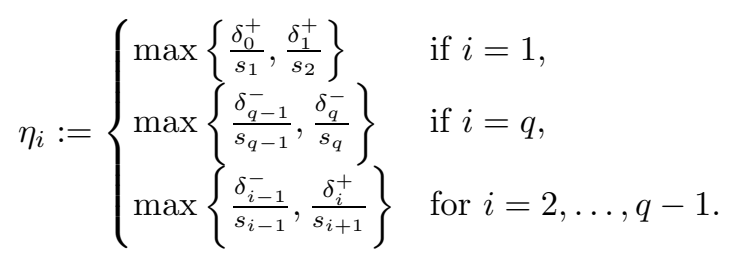

The new Lasota-Yorke inequality is given by the following.

Proposition 3.1 (New Lasota-Yorke inequality). Suppose $\tau \in \mathcal{T}(I)$, i.e., the class of piecewise expanding $\mathcal{C}^{1,1}$ maps on $I$. Then, for every $f \in B V(I)$,

$$
\bigvee_{I} P_{\tau} f \leq \max _{1 \leq i \leq q}\left\{\frac{1}{s_{i}}+\eta_{i}\right\} \bigvee_{I} f+\left[\frac{M}{s^{2}}+\frac{2 \max _{1 \leq i \leq q} \eta_{i}}{\min _{1 \leq i \leq q} m\left(I_{i}\right)}\right] \int_{I}|f| d m
$$


Proof. We will estimate $\bigvee_{I} P_{\tau} f$. Let $0=x_{0}<x_{1}<x_{2}<\cdots<x_{N}=1$ be a partition of $I$. We assume that the points $\tau_{i}\left(a_{i}^{-}\right)$and $\tau_{i}\left(a_{i}^{+}\right), i=1,2, \ldots, q$, are included in this partition. This does not diminish the generality of the considerations. We also assume without loss of generality that

$$
\max \left\{\left|x_{j}-x_{j-1}\right|: j=1,2, \ldots, N\right\} \rightarrow 0 \text { as } N \rightarrow \infty .
$$

Let $I_{i}:=\left(a_{i-1}, a_{i}\right)$. Let us use the following notation:

$$
g_{i}(x):=g\left(\tau_{i}^{-1}(x)\right) \chi_{\tau\left(I_{i}\right)}(x), f_{i}(x):=f\left(\tau_{i}^{-1}(x)\right) \chi_{\tau\left(I_{i}\right)}(x) .
$$

Both functions $g_{i}$ and $f_{i}$ are supported on $\tau\left(I_{i}\right)$.

Let $J_{i}$ denote the set of indices $j$ such that $x_{j-1}$ and $x_{j} \in \tau\left(I_{i}\right)$. We have

$$
\begin{aligned}
& \sum_{j=1}^{N}\left|P_{\tau} f\left(x_{j}\right)-P_{\tau} f\left(x_{j-1}\right)\right| \leq \sum_{j=1}^{N} \sum_{i=1}^{q}\left|g_{i}\left(x_{j}\right) f_{i}\left(x_{j}\right)-g_{i}\left(x_{j-1}\right) f_{i}\left(x_{j-1}\right)\right| \\
\leq & \sum_{i=1}^{q} \sum_{j \in J_{i}}\left|g_{i}\left(x_{j}\right) f_{i}\left(x_{j}\right)-g_{i}\left(x_{j-1}\right) f_{i}\left(x_{j-1}\right)\right| \\
& +\sum_{i=1}^{q}\left(\left|g\left(a_{i-1}^{+}\right) f\left(a_{i-1}^{+}\right) \delta_{i-1}^{+}\right|+\left|g\left(a_{i}^{-}\right) f\left(a_{i}^{-}\right) \delta_{i}^{-}\right|\right) \\
\leq & \sum_{i=1}^{q} \sum_{j \in J_{i}}\left|f_{i}\left(x_{j}\right)\left(g_{i}\left(x_{j}\right)-g_{i}\left(x_{j-1}\right)\right)\right|+\sum_{i=1}^{q} \sum_{j \in J_{i}}\left|g_{i}\left(x_{j-1}\right)\left(f_{i}\left(x_{j}\right)-f_{i}\left(x_{j-1}\right)\right)\right| \\
& +\sum_{i=1}^{q}\left(\frac{\delta_{i-1}^{+}}{s_{i}}\left|f\left(a_{i-1}\right)\right|+\frac{\delta_{i}^{-}}{s_{i}}\left|f\left(a_{i}\right)\right|\right) .
\end{aligned}
$$

Using the Lipschitz condition on $\tau^{\prime}$, we estimate the first sum above as follows:

$$
\begin{aligned}
\sum_{j \in J_{i}}\left|f_{i}\left(x_{j}\right)\left(g_{i}\left(x_{j}\right)-g_{i}\left(x_{j-1}\right)\right)\right| & \leq \sum_{j}\left|f_{i}\left(x_{j}\right) \frac{\tau^{\prime}\left(\tau_{i}^{-1}\left(x_{j-1}\right)\right)-\tau^{\prime}\left(\tau_{i}^{-1}\left(x_{j}\right)\right)}{\tau^{\prime}\left(\tau_{i}^{-1}\left(x_{j}\right)\right) \tau^{\prime}\left(\tau_{i}^{-1}\left(x_{j-1}\right)\right)}\right| \\
& \leq \frac{M}{s^{2}} \sum_{j}\left|f\left(\tau_{i}^{-1}\left(x_{j}\right)\right)\right|\left|\tau_{i}^{-1}\left(x_{j}\right)-\tau_{i}^{-1}\left(x_{j-1}\right)\right| \\
& \leq \frac{M}{s^{2}} \int_{I_{i}}|f| d m+\epsilon_{i}(N) .
\end{aligned}
$$

The last sum above is a Riemann sum, which is estimated by the corresponding integral and an error term $\epsilon_{i}(N)$. It follows from assumption (3.2) that $\epsilon_{i}(N) \rightarrow 0$ as $N \rightarrow \infty$.

Using this estimate,

$$
\begin{aligned}
\sum_{j=1}^{N}\left|P_{\tau} f\left(x_{j}\right)-P_{\tau} f\left(x_{j-1}\right)\right| \leq & \frac{M}{s^{2}} \sum_{i=1}^{q} \int_{I_{i}}|f|+\sum_{i=1}^{q} \epsilon_{i}(N)+\sum_{i=1}^{q} \frac{1}{s_{i}} \bigvee_{I_{i}} f \\
& +\sum_{i=1}^{q}\left(\frac{\delta_{i-1}^{+}}{s_{i}}\left|f\left(a_{i-1}\right)\right|+\frac{\delta_{i}^{-}}{s_{i}}\left|f\left(a_{i}\right)\right|\right) .
\end{aligned}
$$


We divide the last sum into three groups and estimate them as follows:

$$
\begin{aligned}
\frac{\delta_{0}^{+}}{s_{1}}\left|f\left(a_{0}\right)\right|+\frac{\delta_{1}^{+}}{s_{2}}\left|f\left(a_{1}\right)\right| & \leq \max \left\{\frac{\delta_{0}^{+}}{s_{1}}, \frac{\delta_{1}^{+}}{s_{2}}\right\}\left(\bigvee_{I_{1}} f+2 \inf _{I_{1}}|f|\right) \\
& \leq \max \left\{\frac{\delta_{0}^{+}}{s_{1}}, \frac{\delta_{1}^{+}}{s_{2}}\right\}\left(\bar{\bigvee} f+\frac{2}{m\left(I_{1}\right)} \int_{I_{1}}|f|\right) .
\end{aligned}
$$

Similarly,

$$
\frac{\delta_{q-1}^{-}}{s_{q-1}}\left|f\left(a_{q-1}\right)\right|+\frac{\delta_{q}^{-}}{s_{q}}\left|f\left(a_{q}\right)\right| \leq \max \left\{\frac{\delta_{q-1}^{-}}{s_{q-1}}, \frac{\delta_{q}^{-}}{s_{q}}\right\}\left(\bar{\bigvee}_{I_{q}} f+\frac{2}{m\left(I_{q}\right)} \int_{I_{q}}|f|\right) .
$$

Finally, for $i=2, \ldots, q-1$,

$$
\frac{\delta_{i-1}^{-}}{s_{i-1}}\left|f\left(a_{i-1}\right)\right|+\frac{\delta_{i}^{+}}{s_{i+1}}\left|f\left(a_{i}\right)\right| \leq \max \left\{\frac{\delta_{i-1}^{-}}{s_{i-1}}, \frac{\delta_{i}^{+}}{s_{i+1}}\right\}\left(\bigvee_{I_{i}} f+\frac{2}{m\left(I_{i}\right)} \int_{I_{i}}|f|\right) .
$$

Therefore,

$$
\begin{aligned}
\sum_{j=1}^{N} \mid P_{\tau} f\left(x_{j}\right) & -P_{\tau} f\left(x_{j-1}\right)\left|\leq \frac{M}{s^{2}} \sum_{i=1}^{q} \int_{I_{i}}\right| f \mid+\sum_{i=1}^{q} \epsilon_{i}(N)+\sum_{i=1}^{q} \frac{1}{s_{i}} \bigvee_{I_{i}} f \\
& +\max \left\{\frac{\delta_{0}^{+}}{s_{1}}, \frac{\delta_{1}^{+}}{s_{2}}\right\}\left(\bar{\bigvee} f+\frac{2}{m\left(I_{1}\right)} \int_{I_{1}}|f|\right) \\
& +\max \left\{\frac{\delta_{q-1}^{-}}{s_{q-1}}, \frac{\delta_{q}^{-}}{s_{q}}\right\}\left(\bar{\bigvee} f+\frac{2}{m\left(I_{q}\right)} \int_{I_{q}}|f|\right) \\
& +\sum_{i=2}^{q-1} \max \left\{\frac{\delta_{i-1}^{-}}{s_{i-1}}, \frac{\delta_{i}^{+}}{s_{i+1}}\right\}\left(\bar{\bigvee} f+\frac{2}{m\left(I_{i}\right)} \int_{I_{i}}|f|\right) .
\end{aligned}
$$

Estimating $I_{i}$ by $\min _{i} m\left(I_{i}\right)$ and combining appropriate terms together, we get

$$
\begin{aligned}
\sum_{j=1}^{N}\left|P_{\tau} f\left(x_{j}\right)-P_{\tau}\left(x_{j-1}\right)\right| \leq & \max _{1 \leq i \leq q}\left\{\frac{1}{s_{i}}+\eta_{i}\right\} \bigvee_{I} f \\
& +\left[\frac{M}{s^{2}}+\frac{2 \max _{1 \leq i \leq q} \eta_{i}}{\min _{i} m\left(I_{i}\right)}\right] \int_{I}|f| d m+\sum_{i=1}^{q} \epsilon_{i}(N) .
\end{aligned}
$$

Finally, letting $N \rightarrow \infty$, we arrive at inequality (3.1).

Corollary 3.1 ("Standard" Lasota-Yorke inequality). If $s=\min _{1 \leq i \leq q} s_{i}>2$, then we obtain the standard Lasota-Yorke inequality (see [10])

$$
\bigvee_{I} P_{\tau} f \leq 2 s^{-1} \bigvee_{I} f+\left(K+2 \beta^{-1}\right)\|f\|_{\mathcal{L}^{1}},
$$

where $K:=M / s^{2}$ and $\beta:=\min _{1 \leq i \leq q} m\left(I_{i}\right)$. 
Remark 3.1. The classical proof of the standard Lasota-Yorke inequality differs from that of Proposition 3.1 in grouping the terms in the estimates. It uses the inequality

$$
\bigvee_{I} P_{\tau} f \leq \sum_{i=1}^{q}\left(\bigvee_{\tau\left(I_{i}\right)} g_{i} f_{i}+\left|g_{i}\left(\tau\left(a_{i-1}^{+}\right)\right) f_{i}\left(\tau\left(a_{i-1}^{+}\right)\right) \delta_{i-1}^{+}\right|+\left|g_{i}\left(\tau\left(a_{i}^{-}\right)\right) f_{i}\left(\tau\left(a_{i}^{-}\right)\right) \delta_{i}^{-}\right|\right)
$$

and then proceeds similarly as above without mixing the terms from the neighbouring subintervals. This works if $s_{i}>2$ or if both $\delta_{i-1}^{+}$and $\delta_{i}^{-}$are 0 .

The following theorems state conditions under which the coefficient of $\bigvee_{I} f$ in inequality (3.1) is less than 1 . That is,

$$
\max _{1 \leq i \leq q}\left\{\frac{1}{s_{i}}+\eta_{i}\right\} \leq \alpha<1
$$

for some $\alpha>0$.

Theorem 3.2. Suppose $\tau \in \mathcal{T}(I)$ satisfies the following condition:

$$
\frac{1}{s_{i}}+\frac{1}{s_{i+1}} \leq \alpha<1, \text { for } i=1, \ldots, q-1 .
$$

Then (3.3) holds for $\tau$ or for an extension $(\hat{\tau}, \hat{I})$ of $(\tau, I)$ that contains $(\tau, I)$ as an attractor.

Proof. Let us first assume that

$$
\tau(0), \tau(1) \in\{0,1\}
$$

For $i=1$ (and similarly for $i=q)$ :

$$
\frac{1}{s_{1}}+\eta_{1} \leq \frac{1}{s_{1}}+\max \left\{\frac{\delta_{0}^{+}}{s_{1}}, \frac{\delta_{1}^{+}}{s_{2}}\right\} \leq \frac{1}{s_{1}}+\frac{1}{s_{2}} \leq \alpha<1 .
$$

For $i=2, \ldots, q-1$ :

$$
\frac{1}{s_{i}}+\eta_{i} \leq \frac{1}{s_{i}}+\max \left\{\frac{\delta_{i-1}^{-}}{s_{i-1}}, \frac{\delta_{i}^{+}}{s_{i+1}}\right\} \leq \max \left\{\frac{1}{s_{i}}+\frac{1}{s_{i-1}}, \frac{1}{s_{i}}+\frac{1}{s_{i+1}}\right\} \leq \alpha<1 .
$$

If condition (3.4) does not hold, we extend the map $\tau$ to a map $\hat{\tau}$ defined on a larger interval $\hat{I}$ for which condition (3.4) is satisfied and the original system $(\tau, I)$ is an attractor. The idea of the proof is presented in Figure 1.

The following theorem is a generalization of Theorem 3.2 .

Theorem 3.3. Let $s_{i}^{*}:=\frac{s_{i}}{s_{i}-1}$. Suppose $\tau \in \mathcal{T}(I)$ satisfies the following conditions:

(1) For the first branch:
(a) if $\tau\left(a_{0}\right) \notin\{0,1\}$, then $s_{1}>2$;
(b) if $\tau\left(a_{1}^{-}\right) \notin\{0,1\}$, then $s_{2}>s_{1}^{*}$.

(2) For the last branch:

(a) if $\tau\left(a_{q-1}^{+}\right) \notin\{0,1\}$, then $s_{q-1}>s_{q}^{*}$;

(b) if $\tau\left(a_{q}^{-}\right) \notin\{0,1\}$, then $s_{q}>2$.

(3) For all $i=2, \ldots, q-1$ :
(a) if $\tau\left(a_{i-1}^{+}\right) \notin\{0,1\}$, then $s_{i-1}>s_{i}^{*}$;

(b) if $\tau\left(a_{i}^{-}\right) \notin\{0,1\}$, then $s_{i+1}>s_{i}^{*}$.

Then (3.3) holds. 


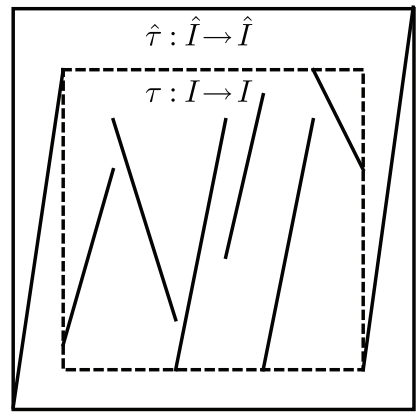

Figure 1. Extending the system $\tau: I \rightarrow I$ to $\tau: \hat{I} \rightarrow \hat{I}$ in such a way that $(\tau, I)$ is the attractor of $(\hat{\tau}, \hat{I})$.

Proof. Let us sketch a proof of (3.3). First, note that we can find $0<\alpha<1$ such that for all $i=1, \ldots, q-1$,

(i) if $s_{i+1}>s_{i}^{*}$, then $s_{i+1} \geq \frac{s_{i}}{\alpha s_{i}-1}>s_{i}^{*}$;

(ii) if $s_{1}>2$, then $s_{1} \geq \frac{2}{\alpha}>2$; and

(iii) if $s_{q}>2$, then $s_{q} \geq \frac{2}{\alpha}>2$.

Now suppose $i=1$. Conditions (ii), (iii) above, and condition (11)(a) of the theorem imply

$$
\frac{1}{s_{1}}+\eta_{1} \leq \max \left\{\frac{2}{s_{1}}, \frac{1}{s_{1}}+\frac{1}{s_{2}}\right\} \leq \alpha<1 .
$$

A similar argument applies when $i=2, \ldots, q$, showing that

$$
\frac{1}{s_{i}}+\eta_{i} \leq \alpha<1
$$

hence proving (3.3).

\section{EXISTENCE AND STABILITY OF ACIM'S}

Our existence and stability results are the applications of known results and methods to a wider space of maps. By a density we mean a function $f \in \mathcal{L}^{1}$ such that $f \geq 0$ and $\int f d m=1$.

Theorem 4.1 (Quasicompactness and existence of acim's). If a map $\tau \in \mathcal{T}(I)$ satisfies inequality (3.1) with the coefficient

$$
\max _{1 \leq i \leq q}\left\{\frac{1}{s_{i}}+\eta_{i}\right\} \leq \alpha<1
$$

for some $\alpha>0$, then for any $f \in B V(I)$ and $n \in \mathbb{N}$,

$$
\left\|P_{\tau}^{n} f\right\|_{B V} \leq \alpha^{n}\|f\|_{B V}+\left(1+\frac{K+2 \beta^{-1}}{1-\alpha}\right)\|f\|_{\mathcal{L}^{1}},
$$

where $K:=M / s^{2}$ and $\beta:=\min _{1 \leq i \leq q} m\left(I_{i}\right)$. Furthermore, $\tau$ admits an acim with a density of bounded variation and where $P_{\tau}: B V(I) \rightarrow B V(I)$ is quasicompact. 
Proof. Using the norm $\|\cdot\|_{B V}:=\bigvee_{I}(\cdot)+\|\cdot\|_{\mathcal{L}^{1}}$ of $B V(I)$ and Proposition 3.1, it follows that for all $n \in \mathbb{N}$,

$$
\left\|P_{\tau}^{n} f\right\|_{B V} \leq \alpha^{n}\|f\|_{B V}+\left(1+\frac{K+2 \beta^{-1}}{1-\alpha}\right)\|f\|_{\mathcal{L}^{1}} .
$$

Since $\left\{f \in B V:\|f\|_{B V} \leq 1\right\}$ is relatively compact in the $\|\cdot\|_{\mathcal{L}^{1}}$ norm, it follows by standard arguments that $P_{\tau}$ has a fixed point in $B V(I)$, the essential spectral radius of $P_{\tau}$ (as defined below) on $B V(I)$ is $\leq \alpha<1$, and $P_{\tau}: B V(I) \rightarrow B V(I)$ is quasicompact.

Definition 4.2 (Essential spectral radius). Consider a bounded linear operator $P$. We denote its spectrum by $\sigma(P)$ and its spectral radius by $r_{\text {spec }}$. The set of all eigenvalues in $\sigma(P)$ that are isolated and of finite multiplicity will be called the discrete spectrum of $P$, denoted by $\sigma_{d i s c}(P)$. The complement of $\sigma_{\text {disc }}(P)$ in $\sigma(P)$ will be called the essential spectrum of $P$, denoted by $\sigma_{e s s}(P)$. The essential spectral radius $r_{e s s}$ of $P$ is defined as the smallest upper bound for all elements of $\sigma_{\text {ess }}(P)$.

Definition 4.3 (Eigenvalue gap, spectral gap). The eigenvalue gap of $P$ is defined as $r_{\text {spec }}-\lambda_{2}$, where $\lambda_{2}:=\sup \left\{\lambda \in \sigma(P):|\lambda|<r_{\text {spec }}(P)\right\}$. The spectral gap of $P$ is defined as $r_{\text {spec }}-r_{\text {ess }}$.

For any $\tau \in \mathcal{T}$, it is well-known that the spectral radius of $P_{\tau}$ on the space $B V(I)$ is equal to 1 . That is, the eigenvalue gap of $P_{\tau}$ equals $1-\lambda_{2}$, while its spectral gap equals $1-r_{\text {ess }}$.

Now we turn to the problem of stability. We shall use the Skorokhod metric as a measure of closeness for maps.

Definition 4.4 (Skorokhod metric). The Skorokhod distance $d_{S}\left(\tau_{\epsilon}, \tau_{0}\right)$ between two maps is the infimum of all positive $r$ such that there exists a subset $A_{r} \subseteq I$ with $m\left(A_{r}\right)>1-r$ and a diffeomorphism $\sigma: I \rightarrow I$ such that

$$
\tau_{\left.\epsilon\right|_{A_{r}}}=\tau_{0} \circ \sigma_{\left.\right|_{A_{r}}},|\sigma(x)-x|<r, \text { and }\left|\frac{1}{\sigma^{\prime}(x)}-1\right|<r,
$$

for all $x \in A_{r}$.

The following stability theorem is a direct consequence of Keller-Liverani stability results (see e.g. Corollaries 1, 2 and Remark 4 of [7) and Proposition 3.1.

Theorem 4.5 (Stability). Consider the one-parameter family of maps $\left\{\tau_{\epsilon}\right\}_{\epsilon \geq 0}$, where $\left\{\tau_{\epsilon}\right\}_{\epsilon \geq 0} \in \mathcal{T}(I)$ uniformly. Suppose there exists $0<\alpha<1$ such that

$$
\max _{1 \leq i \leq q}\left\{\frac{1}{s_{i}}+\eta_{i}\right\} \leq \alpha<1 .
$$

Let $f_{\epsilon}$ be a $\tau_{\epsilon}$-invariant density whose existence is guaranteed by Theorem 4.1. If $d_{S}\left(\tau_{\epsilon}, \tau_{0}\right) \rightarrow 0$ as $\epsilon \rightarrow 0$, then the following statements hold:

(1) The family $\left\{f_{\epsilon}\right\}_{\epsilon>0}$ is relatively compact in $\mathcal{L}^{1}$, and any of its limit functions is a $\tau_{0}$-invariant density.

(2) If $\tau_{0}$ is ergodic, then $\tau_{\epsilon}$ is ergodic for small $\epsilon$ and $f_{\epsilon} \rightarrow f_{0}$ in $\mathcal{L}^{1}$ as $\epsilon \rightarrow 0$ (i.e. $\tau_{0}$ is acim-stable). 
(3) If $\tau_{0}$ is weakly mixing, then the eigenvalue gaps of $\left\{P_{\tau_{\epsilon}}\right\}_{\epsilon}$, for $\epsilon$ small enough, are uniformly bounded, i.e. $0<\gamma<1-\left|\lambda_{2}^{\epsilon}\right|$. As a consequence, there exists a constant $C>0$ such that for all $\epsilon$ small enough and all densities $f \in B V$,

$$
\left\|P_{\tau_{\epsilon}}^{n} f-f_{\epsilon}\right\|_{\mathcal{L}^{1}} \leq C(1-\gamma)^{n}\|f\|_{B V} .
$$

Remark 4.6. It also follows by Theorem 4.1 that there is a uniform spectral gap for the family $\left\{P_{\tau_{\epsilon}}\right\}_{\epsilon \geq 0}$ bounded below by $1-\alpha$.

The stronger L-Y inequality (3.1) allows us to apply the results about stochastic perturbations such as those discussed in [4] and [6] to a wider class of maps satisfying the conditions of Theorems 3.2 or 3.3 . In particular, inequality (3.1) extends the validity of Ulam's approximation method to such maps. Similarly, the results of [12] can be extended to this class of maps.

\section{EXAMPLES}

Below we give examples of situations ensuring that the assumptions of Theorem 4.5 are satisfied.

Example 5.1. Assume that $\tau_{0} \in \mathcal{T}(I)$ and satisfies condition (3.3). Assume that $\left\{\tau_{\epsilon}\right\}_{\epsilon>0}$ is defined on the same partition $\mathcal{P}=\left\{I_{1}, I_{2}, \ldots, I_{q}\right\}$ as $\tau_{0}$, and $\tau_{\epsilon} \rightarrow \tau_{0}$ as $\epsilon \rightarrow 0$ in $\mathcal{C}^{1}\left(\operatorname{int}\left(I_{i}\right)\right)$ for all $i=1,2, \ldots, q$. Then, $d_{S}\left(\tau_{\epsilon}, \tau_{0}\right) \rightarrow 0$ as $\epsilon \rightarrow 0$, $\left\{\tau_{\epsilon}\right\} \subset \mathcal{T}(I)$ uniformly for all $\epsilon \geq 0$, and the conclusions of Theorem 4.5 hold.

Example 5.2. Assume that $\tau_{0} \in \mathcal{T}(I)$ and satisfies condition (3.3). Assume that $\tau_{\epsilon}$ is piecewise expanding on the partition $\mathcal{P}_{\epsilon}=\left\{I_{1}^{(\epsilon)}, I_{2}^{(\epsilon)}, \ldots, I_{q}^{(\epsilon)}\right\}, I_{i}^{(\epsilon)}=\left(a_{i-1}^{(\epsilon)}, a_{i}^{(\epsilon)}\right)$, such that $a_{i}^{(\epsilon)} \rightarrow a_{i}^{(0)}$ as $\epsilon \rightarrow 0$ (in particular, $\tau_{\epsilon}$ has the same number of monotonic branches as $\left.\tau_{0}\right)$. Additionally, assume that there exists $\epsilon_{1}>0$ such that for every $0<\epsilon_{0}<\epsilon_{1}, \tau_{\epsilon} \rightarrow \tau_{0}$ in $\mathcal{C}^{1}$ on the set

$$
\bigcup_{i=1,2, \ldots, q}\left[\max \left\{a_{i-1}^{(0)}, a_{i-1}^{\left(\epsilon_{0}\right)}\right\}, \min \left\{a_{i}^{(0)}, a_{i}^{\left(\epsilon_{0}\right)}\right\}\right]
$$

and that $\left\{\tau_{\epsilon}\right\} \subset \mathcal{T}(I)$ uniformly for all $\epsilon \geq 0$. Then, $d_{S}\left(\tau_{\epsilon}, \tau_{0}\right) \rightarrow 0$ as $\epsilon \rightarrow 0$, and the conclusions of Theorem 4.5 hold.

Example 5.3 (Asymmetric W-map). Let $W_{0}$ be the asymmetric W-map whose graph is shown in Figure 2(a), It is straightforward to check that $W_{0}$ satisfies the slope conditions of Theorem 3.2 . Therefore, it is acim-stable with respect to perturbations described in Examples 5.1 and 5.2

Remark 5.4. W-maps were first constructed by Keller [6] and shown to be acimunstable under perturbations that force the existence of invariant intervals (e.g. perturbations that only move the fixed turning point downward). It was shown in 22 that Markov W-maps could be acim-unstable even with respect to perturbations that do not produce an invariant interval (e.g. perturbations that move the fixed turning point upward). Later in [11] the acim-instability of these maps was proven without assuming that they are Markov. In both of the papers the limiting W-map had slope $=2$ on both sides of the fixed turning point. Recently, in [13] and using the same techniques as [11, it was shown that these maps are acim-unstable (under specific perturbations) if $1 / s_{2}+1 / s_{3} \geq 1$ and acim-stable if $1 / s_{2}+1 / s_{3}<1$. Here $s_{2}$ and $s_{3}$ represent the slopes on the left and right sides of the fixed turning point, respectively. 
Example 5.5. Consider the piecewise linear map $\tau$ with five branches whose graph is shown in Figure 2(b). The partition points are $\left\{0, \frac{1}{20}, \frac{1}{10}, \frac{1}{4}, \frac{1}{3}, 1\right\}$ and the slopes $\left\{16,16, \frac{5}{3}, 3, \frac{3}{2}\right\}$, correspondingly. It is easily checked that $\tau$ satisfies the hypothesis of Theorem 3.3. In fact,

$$
\max _{1 \leq i \leq 5}\left\{\frac{1}{s_{i}}+\eta_{i}\right\}=\max \left\{\frac{1}{16}+\frac{1}{16}, \frac{1}{16}+\frac{3}{5}, \frac{3}{5}+\frac{1}{3}, \frac{1}{3}+\frac{3}{5}, \frac{2}{3}+0\right\}=\frac{14}{15}<1 .
$$

Therefore, $\tau$ is acim-stable with respect to perturbations described in Examples 5.1 and 5.2 ,

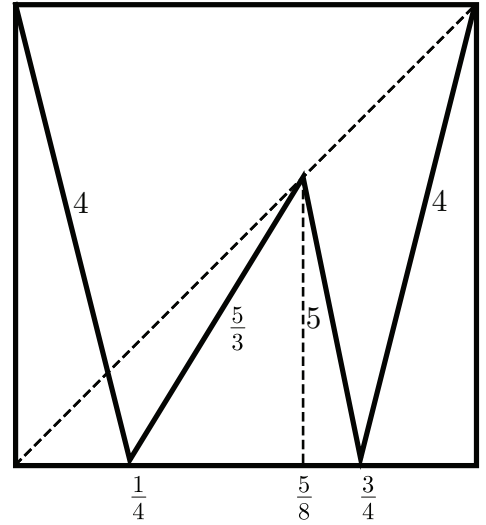

(a)

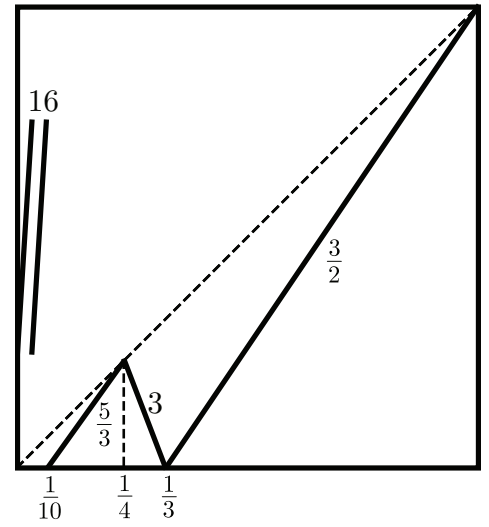

(b)

Figure 2. Figure (a) shows the graph of the asymmetric W-map of Example 5.3. Figure (b) shows the graph of the map $\tau$ of Example 5.5

The results of this paper allow us to answer a question posed in [2].

Example 5.6. Consider the family of unimodal maps $\left\{\tau_{t}\right\}_{0 \leq t<1 / 2}$ defined by

$$
\tau_{t}(x)= \begin{cases}\frac{1}{2}-t+(1+2 t) x, & 0 \leq x<\frac{1}{2}, \\ 2-2 x, & \frac{1}{2} \leq x \leq 1 .\end{cases}
$$

$\tau_{0}$ is exact with invariant density $f_{0}=\frac{2}{3} \chi_{[0,1 / 2]}+\frac{4}{3} \chi_{[1 / 2,1]}$. The question is whether $\tau_{0}$ is acim-stable in the family $\left\{\tau_{t}\right\}_{t \geq 0}$. Note that $\tau_{0}$ has a turning point at $1 / 2$, which is periodic with period 3. Previously known methods did not give an answer to this question.

We will consider the family of third iterates, $\left\{\tau_{t}^{3}\right\}_{t>0}$. The slopes of $\tau_{t}^{3}$ are $s_{1}=s_{3}=s_{7}=2+8 t+8 t^{2}, s_{2}=s_{4}=s_{6}=4+8 t$, and $s_{5}=8$. The map $\tau_{0}^{3}$ is shown in Figure 3(a), and a typical $\tau_{t}^{3}$ is shown in Figure 3(b) Since $\tau_{0}$ is exact, $\tau_{0}^{3}$ is also exact with the same acim; moreover, the acim-stability of $\tau_{0}^{3}$ implies the same for $\tau_{0}$. Because of arbitrarily short intervals in the partitions $\mathcal{P}_{t}$, our results cannot be applied directly to the family $\left\{\tau_{t}^{3}\right\}$. 
Let $g_{1}^{(t)}$ and $g_{7}^{(t)}$ be linear functions which coincide with the first and last branches of $\tau_{t}^{3}$, respectively. For each $t$ we find points $a_{0}^{(t)}$ and $a_{8}^{(t)}$ such that $g_{1}^{(t)}\left(a_{0}^{(t)}\right)=a_{8}^{(t)}$ and $g_{7}^{(t)}\left(a_{8}^{(t)}\right)=a_{0}^{(t)}$. We extend maps $\tau_{t}^{3}$ to $\left[a_{0}^{(t)}, a_{8}^{(t)}\right]$ using the functions $g_{1}^{(t)}$ and $g_{7}^{(t)}$. Let us call the new maps $\hat{\tau}_{t}^{3}$, although they may not be third iterates of some other maps. The new maps are shown in Figure 4(b) for $t>0$ and in Figure 4(a) for the limiting case $t=0$.

The extended family satisfies assumptions of Theorem 3.2 and Example 5.2, so we have acim-stability as described in Theorem 4.5. For all maps $\hat{\tau}_{t}^{3}$ the interval $[0,1]$ is the attractor supporting the unique acim's. Thus, we obtain acim-stability of $\tau_{0}^{3}$ and consequently the acim-stability of $\tau_{0}$.

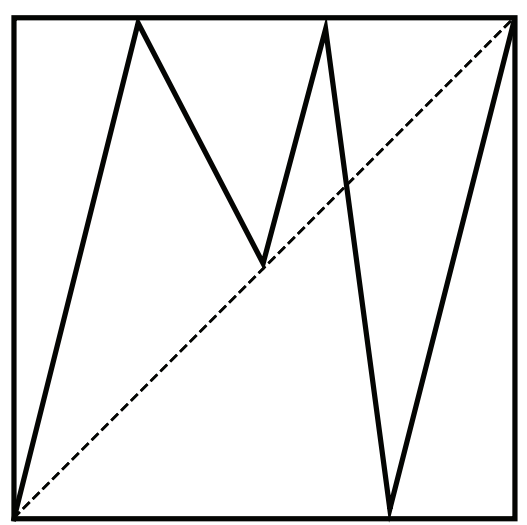

(a)

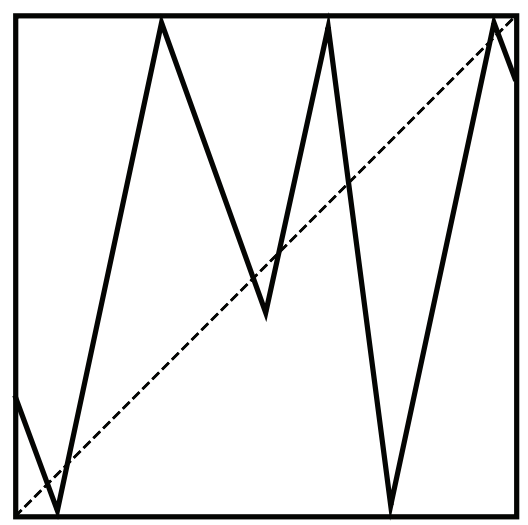

(b)

Figure 3. Figure (a) shows the graph of $\tau_{0}^{3}$ of Example 5.6. Figure (b) shows the graph of $\tau_{t}^{3}$, for $t=0.1$.

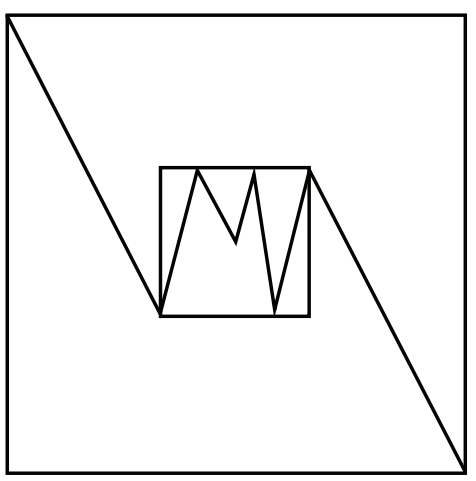

(a)

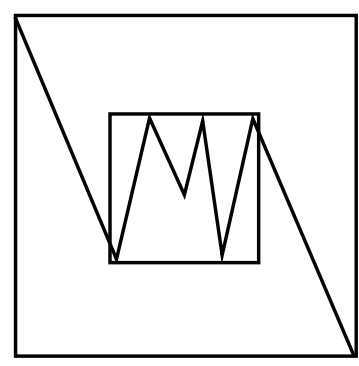

(b)

Figure 4. Figure (a) shows the graph of $\hat{\tau}_{0}^{3}$ of Example 5.6. Figure (b) shows the graph of $\hat{\tau}_{t}^{3}$, for $t=0.05$. 


\section{ACKNOWLEDGMENTS}

The authors thank the anonymous referee for patiently correcting their proofs and for suggesting improvements to the paper. The first author would also like to thank Dr. Oliver Butterley for his valuable remarks on an earlier version of the paper and Professor Carlangelo Liverani for informing the authors of reference [9].

\section{REFERENCES}

1. A. Boyarsky and P. Góra, Laws of Chaos. Invariant Measures and Dynamical Systems in One Dimension, Probability and its Applications, Birkhäuser, Boston, MA, 1997. MR.1461536 (99a:58102)

2. P. Eslami and M. Misiurewicz, Singular limits of absolutely continuous invariant measures for families of transitive maps, J. Difference Equ. Appl., 18 (2012) 739-750. MR2905294

3. E. Giusti, Minimal Surfaces and Functions of Bounded Variations, Birkhäuser, Basel, 1984. MR775682 (87a:58041)

4. P. Góra, On small stochastic perturbations of mappings of the unit interval. Colloq. Math., 49 (1984) 73-85. MR774854 (86f:58135)

5. P. Góra and B. Schmitt, Un exemple de transformation dilatante et $\mathcal{C}^{1}$ par morceaux de l'intervalle, sans probabilité absolument continue invariante, Ergodic Theory and Dynam. Systems, 9 (1989) 101-113. MR.991491 (90h:58052)

6. G. Keller, Stochastic stability in some chaotic dynamical systems, Monatshefte für Mathematik, 94 (4) (1982) 313-333. MR685377 (84k:58130)

7. G. Keller and C. Liverani, Stability of the spectrum for transfer operators, Ann. Scuola Norm. Sup. Pisa Cl. Sci. (4), 28 (1)(1999) 141-152. MR1679080(2000b:47030)

8. Z. S. Kowalski, Continuity of invariant measures for piecewise monotonic transformations. Bull. Acad. Polon. Sci. Sér. Sci. Math. Astronom. Phys., 23 (1975) 519-524. MR.0382593 $(52: 3475)$

9. C. Liverani, Multidimensional expanding maps with singularities: a pedestrian approach. Ergodic Theory and Dynam. Systems, 33 (2013) 168-182. MR3009108

10. A. Lasota and J. A. Yorke, On the existence of invariant measures for piecewise monotonic transformations, Trans. Amer. Math. Soc., 186 (1973) 481-488. MR0335758 (49:538)

11. Zh. Li, P. Gora, A. Boyarsky, H. Proppe and P. Eslami, Family of piecewise expanding maps having singular measure as a limit of ACIM's, Ergodic Theory and Dynam. Systems, 33 (2013) 158-167. MR3009107

12. C. Tokman, B. Hunt and P. Wright, Approximating invariant densities of metastable systems, Ergodic Theory and Dynam. Systems, 31 (2011) 1345-1365. MR2832249

13. Zhenyang Li, W-like maps with various instabilities of acim's, Internat. J. Bifur. Chaos Appl. Sci. Engrg., 23 (2013).

Department of Mathematics \& Statistics, Concordia University, 1455 de Maisonneuve Boulevard West, Montreal, Quebec, Canada H3G 1M8

E-mail address: peslami@mathstat.concordia.ca

Current address: Dipartimento di Matematica, II Università di Roma (Tor Vergata), Via della Ricerca Scientifica, 00133 Roma, Italy

E-mail address: eslami@axp.mat.uniroma2.it

Department of Mathematics \& Statistics, Concordia University, 1455 de Maisonneuve Boulevard West, Montreal, Quebec, Canada H3G 1M8

E-mail address: pgora@mathstat.concordia.ca 